\title{
Design and Dimensioning of Hydrogen Transmission Pipeline Networks
}

\author{
Jean ANDRE ${ }^{1}$, Stéphane AURAY ${ }^{2}$, Jean BRAC ${ }^{3}$, Daniel DE \\ WOLF $^{4}$, Guy MAISONNIER ${ }^{3}$, Mohamed-Mahmoud OULD SIDI ${ }^{5}$, \\ and Antoine SIMONNET 6 \\ ${ }^{1}$ GDF Suez, R\&D Division, Paris, France \\ ${ }^{2}$ CREST-Ensai, EQUIPPE (EA 4476), Université du Littoral, \\ Campus de Ker-Lann, Rue Blaise Pascal, BP 37203, 35172 Bruz \\ cedex, France, Email : stephane.auray@ensai.fr \\ ${ }^{3}$ Institut Francais du Petrole, Rueil-Malmaison, France \\ ${ }^{4}$ TVES (EA 4477), Université du Littoral, Dunkerque, 189B avenue \\ Maurice Schumann, BP 5526, 59379 Dunkerque, France, Email: \\ daniel.dewolf@univ-littoral.fr \\ ${ }^{5}$ INRA, UR1115 Plantes et Systèmes de culture Horticoles, \\ Domaine St. Paul, Site Agroparc, Avignon, F-84914 France, \\ ${ }^{6}$ TOTAL Raffinage Marketing, Paris, France
}

\section{June 17, 2013}

\begin{abstract}
This work considers the problem of the optimal design of an hydrogen transmission network. This design problem includes the topology determination and the pipelines dimensioning problem. We define a local search method that simultaneously looks for the least cost topology of the network and for the optimal diameter of each pipe. These two problems were generally solved separately these last years. The application to the case of development of future hydrogen pipeline networks in France has been conducted at the local, regional and national levels. We compare the proposed approach with another using Tabu search heuristic.
\end{abstract}

Keywords: Hydrogen, energy economics, optimal design, optimal dimensioning.

\section{Introduction}

Taking into account the inevitable exhaustion of fossil fuels and the environmental impact of the emission of greenhouse gases, the actors of the energy sector are engaged in a general reflection to reconcile energy and sustainable development. Hydrogen could play a major role in such reconciliation since it is suggested as future transportation fuel [19]. Unfortunately, The development of this energy 
vector faces numerous technological, economic or social acceptance barriers. According to Johnson et al [19], the lack of major transport infrastructure is one of these major barriers. Indeed, the development of a hydrogen economy will certainly bring important needs for hydrogen delivery solutions from production sites to end users. In a long term vision, these needs are expected to be largely fulfilled by transmission/distribution pipelines. Yet, there is still a lack of consensus on the structure of such a network and its deployment over time. Economic evaluation of hydrogen networks - as well as environmental consideration - is a key criterion to gain a better understanding of these aspects. Therefore, the feasibility study of the long-term deployment of an hydrogen economy was conducted at the European scale (See Castello et al [9]). In France, the Programme d'Action Nationale pour l'Hydrogène (PAN-H) attempts to investigate these challenges. The present work, developed in the framework of the ECOTRANSHY project founded by the PAN-H, aims to develop an economic model for the deployment of hydrogen transmission network for France. The main goals of implementing such a model are to:

- evaluate costs and capacities of all hydrogen transportation means: Trucks (on the short run) versus Pipelines(on the long term)

- study the spatial and temporal development of hydrogen pipeline infrastructure

- study the pipeline infrastructure at various geographic scales: country wide, region wide, city scale.

- develop a software solution integrating those aspects and answering major questions on hydrogen pipeline transmission.

Based on the state of the art in that field, we developed a reliable and transparent network cost model taking into account hydrogen technical constraints. This original model takes into consideration transmission and distribution networks characteristics, operating conditions, energy and material costs. The originality of our work is to consider in a single model, the design problem (the network topology) and the pipe diameter sizing problem. The developed model will be an important piece of the decision tools aiming at designing and planning future hydrogen networks.

The paper is organized as follows. In the next section, we survey the state of the art related to the networks' design and sizing. In section 3, we present our mathematical model in order to determine the optimal characteristics of the network (topology and sizing). A theoretical important result concerning the arborescence topology of the distribution networks by pipelines will be demonstrated. Then, in section 4, we present the proposed approach for the design and sizing of hydrogen networks. Numerical results on local (small), regional, and national networks of refueling stations are presented in section 5 . These results are compared with those obtained using an enumerative method (small networks only) and using the Tabu search algorithm proposed by Blimberg et al [6]. In section 6 we discuss the temporal deployment of hydrogen network before concluding and giving some perspectives for future work in section 7 . 


\section{State of the art}

The literature on the networks design is very rich and covers a large number of fields of application (telecommunications, water, gas, urban transportation, etc.). Graphs are among the most common modeling tools for this purpose. The vertices (nodes) of the graph correspond to the points to be linked (production sites, customers, stations, etc.) and the arcs represent the potential liaisons between vertices (roads, pipelines, cables, etc.). The objective is to ensure the continuity and the quality of the supplied service even in exceptional circumstances. The network design is a very complex problem, especially due to the specifications and constraints to be respected. Indeed, networks have to be reliable, resilient, survivable while ensuring many design constraints (Quality of Service, Cost, etc) [1]. Some instances of the networks design are NP-hard Problems. To deal with such difficult problems, researchers broke them down in many optimization problems (topology, dimensioning, facility location, etc) and tool many simplifying assumptions. For instance, in the classical problem of design of telecommunication networks (See Dolan et al [13])where the capacity of each arc is defined as an upper bound on the flow-rate, the topology of a network can be determined by a minimal spanning tree where the cost of each arc is replaced by its length. This topological result is obtained under several hypotheses e.g. the uniqueness choice for the capacity, not taking into account of the reliability and the resilience. The general problem, without these strong assumptions, is very hard to solve as discussed in the survey on the survivability of telecommunications networks proposed by Kevin and Mahjoub in [21].

On the pipe networks (water, natural gas, hydrogen), the capacities are given by the nonlinear relations linking the flow and the pressures at both extremities of the pipe. The first work on pipeline networks design, to the best of our knowledge, was done by Rothfarb et al [32] and aimed at optimally collecting gas productions from a set of offshore wells. In Bhaskaran et al [7], the authors are confronted with a similar problem of optimal design of a network of collection of several wells in a desert environment (Australia). They show that, under certain conditions, the optimal collecting network is a treelike network. Note that both works of Rothfarb et al [32] and Bhaskaran et al [7] consider only networks of collection of gas from several wells (multi-sources) but with a unique collection point. With this restriction, the value of the flow on each arc is fixed. In Walters [36], the author uses the techniques of the dynamic programming to investigate all the possible trees on a water distribution network with several sources (springs) and the multiple wells (with pressures fixed to sources and minimal pressures at the points of exits). Similarly, further work uses artificial intelligence techniques. Thus, Nie [35] deals with the optimal topology of pipes networks with cycles and multi-sources using neural networks. De la Cruz et al [10] used an evolutionary multiobjective constrained optimization algorithm to design optimal distribution of petroleum products through oil pipelines networks. Brimberg and al. [8] consider the south Gabon oil fields to design a collection network from a given set of offshore platforms to a given port. The network topology and discrete sizes of pipes must be chosen to minimize construction costs. This problem was expressed as a mixed-integer program, and solved both heuristically by Tabu Search and Variable Neighborhood Search methods and exactly by a branchand-bound method. Donkoh et al [14] combined factor rating method with Prim 
and Steiner tree algorithms and geometry to design optimal pipeline network in the framework of West African Gas Pipeline Project. They claim that their proposed solution is topologically equivalent to the existing network and hence optimal in pipes lengths and project cost.

More recently, R.S. Middleton and J.M. Bielicki [25] develop SimCSS; a scalable infrastructure model; for the design of pipelines network connecting carbon sources and storage reservoirs. The authors formulate the problem as a mix integer linear program (MILP) and use ILOG's CPLEX 11.0 mixed integer optimizer to solve it. Note that the SimCSS model doesn't take into consideration the nonlinear relations between pipe diameters and gas flows. It also assumes that the objective function is linear by considering only fixed construction costs. Johnson and Ogden [19] present the HyPAT, a spatially-explicit, model for optimizing a long-term hydrogen pipeline. The objective is to identify the optimal infrastructure design for producing $\mathrm{H}_{2}$ and connecting production facilities to demand centers. The problem is formulated as a MILP and solved using General Algebraic Modeling System (GAMS). This model has the same restrictions as SimCSS i.e. the linear objective function and the omission of the nonlinear relations between pipe diameters and gas flows. In industrial countries, constraints on laying pipelines are more and more taken into account by researchers. Therefore, the dimensioning of pipelines with fixed topology is more considered in the optimal design of networks. Osiadack and Gorecki [28] and De Wolf and Smeers [11] used the bundle method that gave good results for a new trunk line of the Belgian network. Zhang and Zhu [39] took into account the discrete aspect of the commercial diameters by proposing the distribution of the sizes on every section. Similarly, Babonneau and Vial [3] deal with the design of the water networks. Finally, the reinforcement problems of existing networks (by doubling some pipes) appeared with the work of André et al [2].

In perspective studies dealing with future large-scale hydrogen economy, the total length of potential network is often estimated based on data from the existing natural gas network and aggregated by consumer. Then, the cost is estimated by multiplying the total length by a unit distance price. The studies of Castello et al [9] on the French case and of Smit et al [33] on the Dutch case are examples among others. Other models based on geographical information systems (GIS), estimate the real distances between production and consumption points located on the map. The search for a topology is reduced to the search for a set of pipelines leaving the source. The MOREHyS model developed and applied for the German case by Ball [4] falls in this category. This approach tends to overestimate the construction since it cannot take into account the economy of the aggregation of several demands in the same pipeline. Intermediate models estimate the length of the network by assuming its layout as known. For example, Yang et al [37] consider a system of concentric rosettes in an ideal American city. More detailed models are using minimal spanning algorithms to optimize the network's total length. Lin et al [15] justify this choice by indicating that the total length of the pipeline so obtained is the most significant factor of the transportation costs. Also, Patay et al [31] use the same techniques. All these approaches suppose that the pipelines have the same diameter on all the sections of a network of the same level: national transport, regional transport, local distribution. This standardization of diameters allows to estimate average costs by distance unit 
i.e. $€ /$ kilometer (See Smit et al [33]).

Based on this state of the art, this paper aims to go further on both aspects of the design and dimensioning of networks of pipeline of hydrogen. On the one hand, we wish to go beyond the simple algorithms of minimal spanning to elaborate a method determining an optimal topology dedicated to pipelines. On the other hand, we wish to overcome the simplification of the standardization of diameters by proposing a method adjusting diameters by section in order to reduce the costs. The third objective is to couple both optimizations topology / dimensioning which are strongly connected.

\section{Mathematical developments}

\subsection{Mathematical model}

In this section, we first introduce some useful notations used in this paper. We then formulate the design problem we deal with as a constrained nonlinear optimization program. The network is modeled by means of a graph and consists in a set of nodes and arcs. We note by $N$ the set of all hydrogen supply and gas consumption nodes and by $A$ the set of arcs of the network. We note by $s_{i}$ the gas supply at node $i$ and by $d_{i}$ the as demand at node $i$. The length and the diameter of the pipe linking node $i$ to node $j$ will be respectively noted $\mathrm{Lij}$ and $D_{i j}$. The hydrogen flow in this pipe is noted $Q_{i j}$. Finally, we note by $\pi_{i}$ the square of the gas pressure at node $i$, and by $\pi_{\min }$ and $\pi_{\max }$, the minimal and the maximal values of $\pi_{i}$. The inputs of the developed model are the geographical coordinates of the node set, hydrogen demand and supply at each node, are the minimal and maximal pressures required at each node.

Note that for the problem of the determination of the optimal topology, this set is determined by the model. These quantities are also given in our model. Our objective here is to find a set of connected arcs $A \subset N \times N$ and to identify the pipes diameters $D_{i j} \forall(i, j) \in A$ such that the total cost of the resulting network is minimal and some constraints are satisfied. Therefore, we mathematically formulate the problem of optimal design and sizing of a hydrogen transportation network as follows:

$$
\begin{aligned}
& \min C(\pi, D, Q)=\sum_{(i, j) \in A}\left(a_{0}+a_{1} D_{i j}+a_{2} D_{i j}^{2}\right) L_{i j} \\
& \text { subject to }\left\{\begin{array}{l}
\pi_{i}-\pi_{j}=k^{\prime} Q_{i j}^{2} \frac{L_{i j}}{D_{i j}^{5}}, \forall(i, j) \in A \\
s_{i}+\sum_{k \mid(k, i) \in A} Q_{k i}=\sum_{j \mid(i, j) \in A} Q_{i j}+d_{i}, \forall i \in N \\
D_{\min } \leq D_{i j} \leq D_{\max }, \forall(i, j) \in A \\
\pi_{\min } \leq \pi_{i} \leq \pi_{\max }, \forall i \in N
\end{array}\right.
\end{aligned}
$$

The objective function $C$ is sum of the costs of hydrogen transportation pipelines. The costs of a gas pipeline could be divided into capital expenditures 
(Capex) and operating costs (Opex). Capital expenditures, widely dominating, include two main items: the material costs and the installation costs. The operating costs are considered as a percentage of the capital cost. This is the reason why we use, as an optimization criterion, only the pipe investment costs. As for the hydrogen, the use of the natural gas economic models is generally agreed by economists (See Castello et al [9] and Parker [30]). The costs functions linking diameters $\mathrm{D}(\mathrm{mm})$ to the costs by units of length $(€ / \mathrm{km})$ for natural gas available in the literature could be linear (See Castello [9]), however the quadratic models are the most used (See De Wolf and Smeers [11], Hafner[17] or Parker [30]). Therefore,in this study we adopt the quadratic costs function $C\left(D_{i j}\right)$ :

$$
C\left(D_{i j}\right)=a_{0}+a_{1} D_{i j}+a_{2} D_{i j}^{2}
$$

The first constraint is related to the pressure drop equation for the hydrogen. When we consider a fluid flowing in a pipeline, the difference of pressure between two ends of the pipe finds its origin in the friction of the fluid on the internal wall of the pipe. These energy losses, called head losses (See Joulié [20]), depend on physical properties of the fluid (density, viscosity) and on the geometry of the pipe (diameter, length and roughness).The literature concerning the head losses proposes several formulae which differ according to the required degree of accuracy. For the present work, we have used the equation used by De Wolf and Smeers [11] linking the gas flow $Q_{i j}\left(\mathrm{~m}^{3} /\right.$ hour $)$, the pressure at the entry of the pipe $p_{i}$ (bar) and the pressure at the exit of the pipe $p_{j}$ (bar):

$$
Q_{i j}=K\left(D_{i j}\right) \sqrt{p_{i}^{2}-p_{j}^{2}}, \quad \forall(i, j) \in A
$$

where the coefficient $K\left(D_{i j}\right)$ can be computed by the following formula:

$$
K\left(D_{i j}\right)=0.0129 \sqrt{\frac{D_{i j}^{5}}{\lambda \cdot Z_{m} \cdot T_{m} \cdot L_{i j} \cdot d}}
$$

with $D_{i j}=$ the pipe diameter $(\mathrm{mm})$,

$\lambda=$ the dimensionless coefficient of friction,

$Z_{m}=$ the dimensionless compressibility factor,

$T_{m}=$ the gas mean temperature (Kelvin),

$L_{i j}=$ the pipe diameter $(\mathrm{km})$,

$d=$ the relative density of the gas with regard to the air.

This equation can be rewritten in the following way:

$$
\pi_{i}-\pi_{j}=k^{\prime} Q_{i j}^{2} \frac{L_{i j}}{D_{i j}^{5}}, \forall(i, j) \in A
$$

since we have defined $\pi_{i}$ as the square ratio of pressure at the entry of the pipe, and $\pi_{j}$, as the square ratio of pressure at the exit of the pipe.

The second constraint expresses the node flow conservation equations. The third constraint gives the accepted minimal and maximal pressures at a given node. The fourth constraint is related to the pipes' sizes available on the market.

The mathematical program (1) is an integer program due to the binary choice of opening the arcs (the choice of $A \subset N \times N$ ) and also nonlinear due to the 
pressure drop constraints. This kind of optimization programs is difficult to be solved using exact methods. Therefore, in this paper we will use a heuristic to solve this problem.

Note that we only consider continuous diameters while other models (R.S. Middleton and J.M. Bielicki [25] or Johnson an Ogden [19]) consider discrete values for the pipe diameters. This choice has two motivations:

- First, today there is no clear idea about the commercial diameters for hydrogen that we will have tomorrow. Note that, the additional cost due to the difference between proposed and available diameters could be easily estimated.

- Second, our model is only a first approach to estimate the cost of development of a new hydrogen network. This estimation could be better adjusted with future studies.

\subsection{Characteristics of the optimal topology}

In appendix A, using the work of Bhaskaran and al. [7], we demonstrate that with the choice made for the investment objective function and for the head losses equation, the optimal networks are trees.

Lemma. With a quadratic cost function (2) and with the head losses equation (3), the optimal structure of the network is a tree.

Proof: See appendix A.

\section{Proposed approach}

In this section, we introduce the general approach developed for the design and the sizing of a transportation or distribution hydrogen network. The general approach relies on two main subroutines namely: the minimal-length spanning tree to determine the initial topology and the optimal continuous sizing of the diameters on a given tree. After the initial point determination, various local search methods are explored to improve the combined topology/sizing solutions.

The first step is the initialization of the topology. The objective is to determine the topology with the minimal length of the considered network. Taking advantage of the result indicating that the optimal network is treelike, we use a classical algorithm of determination of the minimal spanning tree (See Bang et al [5]). The input data of this subproblem are the geographical coordinates of the nodes of the network to calculate the intra-node distances.

The second step performs the sizing of the continuous diameters on a given tree. For a fixed treelike topology of the network, this module determines the optimal sizing of the diameters. Hence, the model (1) is reduced to the following 
program:

$$
\begin{aligned}
\min C(\pi, D)= & \sum_{(i, j) \in T}\left(a_{0}+a_{1} D_{i j}+a_{2} D_{i j}^{2}\right) \cdot L_{i j} \\
\text { s.t. } & \left\{\begin{array}{l}
\pi_{i}-\pi_{j}=k^{\prime} Q_{i j}^{2} \frac{L_{i j}}{D_{i j}^{5}}, \forall(i, j) \in T \\
s_{i}+\sum_{k \mid(k, i) \in A} Q_{k i}=\sum_{j \mid(i, j) \in A} Q_{i j}+d_{i}, \forall i \in N \\
D_{\text {min }} \leq D_{i j} \leq D_{\max }, \forall(i, j) \in T \\
\pi_{\text {min }} \leq \pi_{i} \leq \pi_{\text {max }}, \forall i \in N
\end{array}\right.
\end{aligned}
$$

Let us note that the set $A$ is reduced to a tree $T$ and flows $Q_{i j}$ are not any more decision variables since these variables can be infered from the treelike structure. This program, although no longer combinatorial, is strongly nonlinear and nonconvex because of the presence of the pressure equations. It has been solved using the nonlinear solver SNOPT developed by Stanford University (See Gill et al [15]) and available within the TOMLAB environment. Let us note that this solver only supplies local optima when the program is non-convex. The output of this program consists of a list of optimal diameters minimizing the total cost and satisfying the aforementioned constraints.

The third step regards the topology/sizing optimization with different solution approaches. The first one is the Brute Force approach or enumerative approach that can be performed on small scale problems. This method has the advantage to identify the optimal solutions by enumeration of all possible trees (with the recomputation of the optimal diameter for each new tree). The total number of trees was computed using Cayley's formula giving the number of spanning trees of a complete graph with $\mathrm{n}$ nodes by $n^{n-2}$. The selected algorithm is available in the references of Kapoor [24] and Minty [27].

As the scale of the problems increases, the complexity significantly increases and the enumerative method reaches its limits with regards to the tractability. Heuristics have been then investigated to cope with this tractability problem in order to reach a local solution. The Delta change, is a heuristic that tries to improve the cost of the treelike network by applying local modifications. This algorithm is inspired from Rothfarb and al. [32]. This heuristic tries to decrease the cost of the network by making local changes on arcs, and thus on the topology. Table 1 gives the pseudo-code of the delta change subroutine and Figure 1 illustrates it on a simple example.

Note that, two main modifications were introduced into the delta-change algorithm in order to improve its performance. First, contrary to the approach of Rothfarb et al. [17], the assessment for the cost of each tree is done using the SNOPT nonlinear solver and not using dynamic programming. Second, we randomly investigate the nodes and not only from the closest to the furthest like in the original version. Therefore, in order to evaluate the quality of the solution of this heuristic method, we opted for the following strategy:

- For the small networks, we compared the delta-change heuristic with an enumerative one finding the global optimum [24],[27]. 
Step 0: Start from an initial minimal spanning tree with its corresponding cost (using SNOPT solver).

Step 1: Sort the nodes for exploration either on a distance-to-source criterion or randomly. Select a subset of nodes or the total set of nodes.

Step 2: For each selected exploration node $n_{i}$ of the tree:

Step 2a: Select the closest nodes $n_{i, j}$ (in Euclidian distance) not connected with an arc from the tree to $n_{i}$.

Step 2b: For each $n_{i, j}$, add the arc $\left(n_{i}, n_{i, j}\right)$ and determine the cycle so created.

Step 2c: Remove one by one the other arcs of the cycle.

Step 2d: As soon as the cost is improved, the new network replaced the previous best network.

Step 3: Repeat the previous steps until a stopping criterion is reached.

Table 1: Delta change subroutine

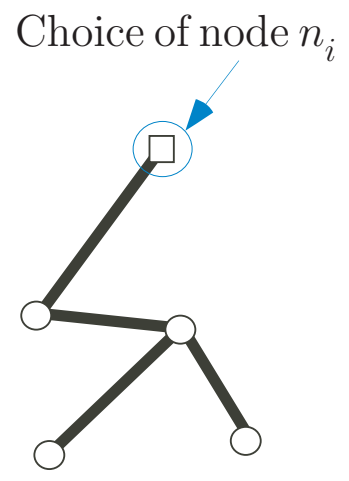

Selection of closest node $n_{i, j}$
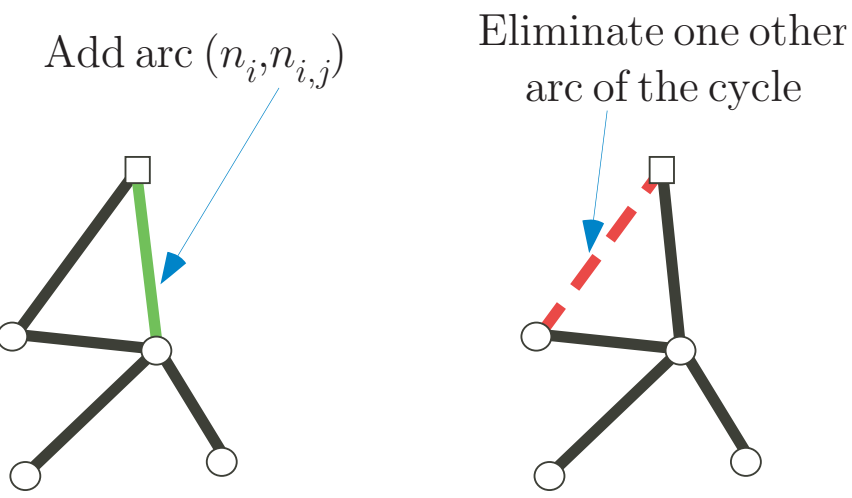

Figure 1: Illustration of the Delta change 
- For the large networks, we compared the results obtained using the deltachange algorithm with those of the Tabu search algorithm proposed in Brimberg et al [8]. According to authors, the Tabu search is able to find near optimal solutions, i.e. with small deviation from the optimal solution and for a large range of test problems.

The Tabu Search principles are based on the systemic exploration of a Tabu list in the hope to find other local optima that might be better than the current local optimum without any chance to come back to previous choice.

The pseudo code of the Tabu Search proposed by Brimberg et al. is given in Table 2 .

Step 0: Initialize the topology with the minimal spanning tree and the initial sizing solution (local search method using SNOPT solver) and initialize the Tabu list (empty list with a predefined length).

Step 1: Sort the nodes for exploration either on a distance-to-source criterion or randomly. Select a subset of nodes or the total set of nodes.

Step 2: For each selected exploration node $n_{i}$ of the tree:

Step 2a: Select the closest nodes $n_{i, j}$ (in Euclidian distance) not connected with an arc from the tree to $n_{i}$.

Step 2b: Determine the set of all arcs that do not belong to the current tree and not in the Tabu list (empty at the first iteration).

Step 2c: For each of these arcs, determine the cycle created when adding the arc to the initial tree. Delete in turn one by one others arcs of the cycle, update the flows in the remaining arcs and compute the resulting costs.

Step 2d: From all elementary tree transformations above, keep the pair of added and deleted arcs with the best cost (the elementary transformation for which the cost decreases the most or increases the least).

Step 3: Apply the corresponding exchange to obtain the new current solution.

Step 4: Set a Tabu restriction on the reverse move of the leaving and entering arcs, i.e. update Tabu list. If an improved solution has been found, it becomes the new incumbent.

Step 5: Repeat the preceding steps until a stopping criterion is reached.

Table 2: Pseudo code of the Tabu Search

The reader will notice that the proposed Delta Change and the Tabu Search algorithms are based on the same principles of generating cycles and investigation. However, one main difference between these algorithms is that the delta change switches to another node as soon as a better solution is found while the tabu search explores all the transformations within the cycles before selecting the best one in the hope of finding a better solution. Note that tabu search could select one solution increasing the cost if all elementary transformations lead to increasing costs. 


\section{Numerical results}

In this section, we first illustrate our design approach on two small networks. For each small network, we identified the global optimal solution (topology, diameters' sizes, cost) using an enumerative method. We compare the optimal solutions with those obtained using the delta-change and Tabu search heuristics. Then, we compare the performances of the two heuristics. Third, we consider a real (regional) hydrogen network and we compare the performances of the two heuristics. Finally, we apply the delta-change heuristic to the design of a future French national hydrogen network.

Before going into more details, we provide in this section some elements about the parameters' setting for each heuristic. We performed simulations for different percentages of nodes investigation ( $\%$ of investigated nodes). Note that, the number of nodes $n_{i}$ to be investigated is the result of the multiplication of this percentage by the network size $N$ (number of network nodes) and we consider four investigation rates $(5 \%, 10 \%, 50 \%$, and $100 \%)$. For each investigation percentage, we considered $2,3,4,5$, and 6 neighbors to be visited i.e. not directly connected to the current investigated node. The multiplication of the number of nodes to be investigated by the number of neighbors gives the number of cycles created for each simulation. The number of arcs explored by cycles, however, depends on the cycles topologies and can not be a priori estimated.

Simulations were performed using two investigation strategies for $n_{i}$. First, we explored nodes from the closest to the furthest as suggested by the authors of delta-change. Then, we considered a random exploration of the nodes. In this last case, simulations were repeated 5 to 10 times, depending on the network size, to take into account the stochastic aspects of our choice. The stopping criterion considered for the delta-change is the maximum number of created cycles which is equal to the number of the visited nodes multiplied by the number of the non connected (neighbors) nodes considered. In addition of this stopping criterion, the Tabu search stops when the maximal length of the Tabu list is reached. Note that, we don't use the computational time as a stopping criterion, as done by Brimberg, in order to assess the quality of solutions obtained by each algorithm. This choice is also motivated by the Tabu list management step which could be time consuming and thus could handicap this algorithm. The maximum length of the Tabu List in our simulations was set to 20 ( 7 was the value used by Brimberg et al [8]).

\section{$5.1 \quad$ Test examples}

Two small networks were first built to demonstrate the gain of the delta change heuristic with respect to a minimal spanning tree. The two test examples concern a set of consumption nodes (nodes 1 to 6 ) provided by a single hydrogen plant (node 7 ). The pressure at the exit of the hydrogen plant is $40 \mathrm{bar}$ and the required pressure at the demand nodes is 36 bar. The demands of hydrogen are identical on all the consumption points $\left(47,214 \mathrm{~m}^{3}\right.$ per day). Two different geographical configurations were tested:

- Test network 1 where consumption nodes form square. 
- Test network 2 where consumption nodes form a rectangle.

For the two small networks, we first used the delta-change and Tabu Search heuristics following the parameters setting explained above. However, we decided to explore all nodes (100\% of nodes investigated) and we tried to explore the maximum numbers of no connected nodes ( 6 neighbors if possible) for the random exploration strategy. This choice is due to the necessity to repeat simulations for this choice. The total number of trees is of 16,807 ( $7^{5}$ trees) for each case.

\subsubsection{Test Network 1}

The first test network is illustrated on Figure 2. Results obtained by heuristics (delta-change and Tabu Search) are given and commented below. Table 3 presents the results when nodes are ordered from the closest to the furthest. Table 4 presents the results when nodes are randomly ordered. The simulations were repeated 10 times for this last case.

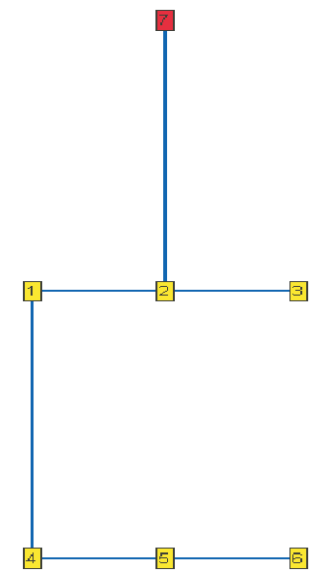

a) Minimal spanning tree

Length $=182.4 \mathrm{~km}$, Cost $=7,365,756 €$

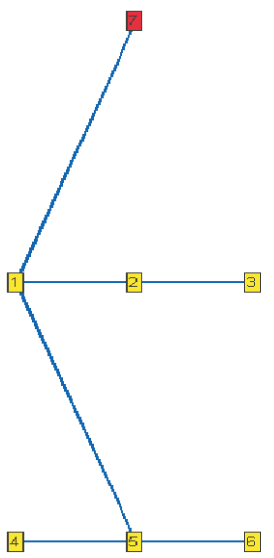

b) Tree after delta change

(from the closest to the furthest strategy) Length $=187.9 \mathrm{~km}$, Cost $=6,868,264 €$

Figure 2: test network 1

\begin{tabular}{|c|c|c|c|c|c|}
\hline Visited & \% of investigated & \multicolumn{2}{|c|}{ DC } & \multicolumn{2}{c|}{ TS } \\
\cline { 3 - 6 } nodes $n_{i, j}$ & nodes $n_{i}$ & Cost $(€)$ & CPU (s) & Cost $(€)$ & CPU (s) \\
\hline 2 & 100 & $6,868,264$ & 103.78 & $6,867,704$ & 74.10 \\
\hline 3 & 100 & $6,868,264$ & 133.21 & $6,867,704$ & 114.05 \\
\hline 4 & 100 & $6,868,264$ & 174.85 & $6,867,704$ & 126.18 \\
\hline 5 & 100 & $6,868,264$ & 190.51 & $6,867,704$ & 123.59 \\
\hline 6 & 100 & $6,868,264$ & 190.52 & $6,867,704$ & 124.18 \\
\hline \multicolumn{2}{|c|}{ Average } & $6,868,264$ & 158.58 & $6,867,704$ & 112.42 \\
\hline
\end{tabular}

Table 3: Results for test network 1 when nodes are ordered from the closest to the furthest 


\begin{tabular}{|c|c|c|c|c|c|c|}
\hline \multirow{2}{*}{ Run } & Visited & \% of investigated & \multicolumn{2}{|c|}{ DC } & \multicolumn{2}{c|}{ TS } \\
\cline { 3 - 7 } & nodes $n_{i, j}$ & nodes $n_{i}$ & Cost $(€)$ & CPU (s) & Cost $(€)$ & CPU (s) \\
\hline 1 & 6 & 100 & $\mathbf{6 , 3 3 0 , 1 1 9}$ & 140.60 & $\mathbf{6 , 3 3 0 , 1 1 9}$ & 169.40 \\
\hline 2 & 6 & 100 & $\mathbf{6 , 3 3 0 , 1 1 9}$ & 133.89 & $6,867,704$ & 150.05 \\
\hline 3 & 6 & 100 & $\mathbf{6 , 3 3 0 , 1 1 9}$ & 132.99 & $6,867,704$ & 138.54 \\
\hline 4 & 6 & 100 & $\mathbf{6 , 3 3 0 , 1 1 9}$ & 182.57 & $\mathbf{6 , 3 3 0 , 1 1 9}$ & 168.60 \\
\hline 5 & 6 & 100 & $\mathbf{6 , 3 3 0 , 1 1 9}$ & 144.19 & $\mathbf{6 , 3 3 0 , 1 1 9}$ & 154.85 \\
\hline 6 & 6 & 100 & $6,868,264$ & 164.53 & $6,867,704$ & 144.30 \\
\hline 7 & 6 & 100 & $7,052,000$ & 190.46 & $6,330,119$ & 152.47 \\
\hline 8 & 6 & 100 & $6,867,704$ & 184.09 & $6,867,704$ & 136.14 \\
\hline 9 & 6 & 100 & $6,867,704$ & 157.12 & $6,867,704$ & 140.57 \\
\hline 10 & 6 & 100 & $6,867,704$ & 151.75 & $6,330,119$ & 164.20 \\
\hline \multicolumn{2}{|c|}{ Average } & $\mathbf{6 , 6 1 7 , 3 9 7}$ & $\mathbf{1 5 8 . 2 2}$ & $\mathbf{6 , 5 9 8 , 9 1 2}$ & $\mathbf{1 5 1 . 9 2}$ \\
\hline
\end{tabular}

Table 4: Results for test network 1 when nodes are randomly ordered

The optimal solution identified by enumerative method corresponds to the cost of $\mathbf{6 , 3 3 0 , 1 1 9} \in$ and to more than 50,000 seconds of computational time. As we can observe in Table 3, for the determinist exploration strategy, both algorithms were not able to identify the optimal solution. The mean deviation from the optimal for the delta-change and Tabu Search is roughly the same (8.5\%). The mean (average) values indicate that Tabu search obtained slightly better solutions than delta-change. As we can note in Table 4, each heuristic has obtained better results with the optimal solution reached 5 times for the DC and 3 times for the TS out of 10 runs for the random exploration strategy. Using this strategy, the mean deviations from the optimal solution for the delta-change and Tabu Search are respectively of $4.5 \%$ and $4.2 \%$. However, the difference between the two solutions is very small (only hundreds of $€$ ). Regarding the computational times, the delta-change heuristic is slightly slower on average compared to the Tabu search.

\subsubsection{Test network 2}

The second test network is illustrated on Figure 3. Table 5 presents the results for the second test network when nodes are ordered from the closest to the furthest. Table 6 presents the results for the second test network when nodes are randomly ordered.

For this test network, the optimal solution corresponds to the cost of $\mathbf{8 , 0 3 0 , 9 8 1} €$ and its computational time is roughly the same as for the first network. As for the first network, the determinist exploration strategy didn't lead to identify the optimal solution and that holds using both algorithms. The mean deviations from the optimal solution for the delta-change and Tabu Search are respectively of $3.6 \%$ and $8.1 \%$ using this exploration strategy. Thus, the deltachange obtained better solutions than Tabu search for all the runs. Regarding the computational times, the delta-change heuristic is the slowest algorithm compared to the Tabu search. The delta-change and Tabu Search have respectively identified the optimal solution 3 and 4 times within the 10 runs for the random 


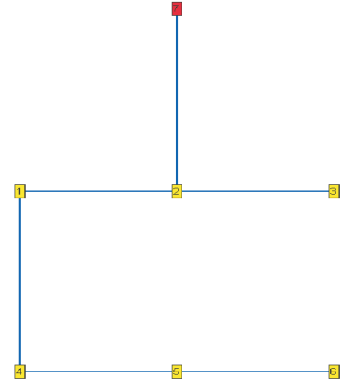

a) Minimal spanning tree

Length $=253.4 \mathrm{~km}$, Cost $=10,149,111 €$

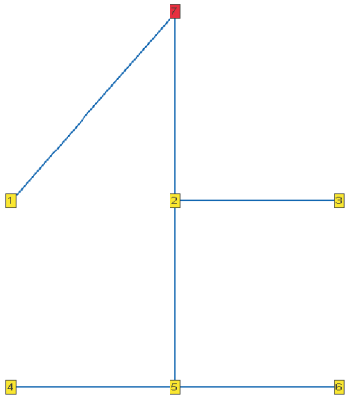

b) Tree after delta change (from the closest to the furthest strategy) Length $=283.8 \mathrm{~km}$, Cost $=8,316,807 €$

Figure 3: test network 2

\begin{tabular}{|c|c|c|c|c|c|}
\hline Visited & \% of investigated & \multicolumn{2}{|c|}{ DC } & \multicolumn{2}{|c|}{ TS } \\
\cline { 3 - 6 } nodes $n_{i, j}$ & nodes $n_{i}$ & Cost $(€)$ & CPU (s) & Cost $(€)$ & CPU (s) \\
\hline 2 & 100 & $8,316,807$ & 78.24 & $9,102,252$ & 45.41 \\
\hline 3 & 100 & $8,316,807$ & 93.83 & $8,578,927$ & 97.14 \\
\hline 4 & 100 & $8,316,807$ & 113.80 & $8,578,927$ & 99.14 \\
\hline 5 & 100 & $8,316,807$ & 137.73 & $8,578,927$ & 127.72 \\
\hline 6 & 100 & $8,316,807$ & 138.07 & $8,578,927$ & 127.87 \\
\hline \multicolumn{2}{|c|}{ Average } & $8,316,807$ & 112.33 & $8,683,592$ & 99.46 \\
\hline
\end{tabular}

Table 5: Results for test network 2 when nodes are ordered from the closest to the furthest

\begin{tabular}{|c|c|c|c|c|c|c|}
\hline \multirow{2}{*}{ Runs } & Visited & \% of investigated & \multicolumn{2}{|c|}{ DC } & \multicolumn{2}{c|}{ TS } \\
\cline { 3 - 7 } & nodes $n_{i, j}$ & nodes $n_{i}$ & Cost $(€)$ & CPU (s) & Cost $(€)$ & CPU (s) \\
\hline 1 & 6 & 100 & $8,108,526$ & 80.13 & $\mathbf{8 , 0 3 0 , 9 8 1}$ & 138 \\
\hline 2 & 6 & 100 & $8,108,527$ & 100.59 & $8,931,651$ & 149.6 \\
\hline 3 & 6 & 100 & $9,522,883$ & 129.48 & $\mathbf{8 , 0 3 0 , 9 8 1}$ & 133.03 \\
\hline 4 & 6 & 100 & $9,222,245$ & 102.54 & $8,104,990$ & 136.19 \\
\hline 5 & 6 & 100 & 8583925 & 54.41 & $8,856,696$ & 160.38 \\
\hline 6 & 6 & 100 & $\mathbf{8 , 0 3 0 , 9 8 1}$ & 64.44 & $\mathbf{8 , 0 3 0 , 9 8 1}$ & 132.32 \\
\hline 7 & 6 & 100 & $\mathbf{8 , 0 3 0 , 9 8 1}$ & 126 & $8,390,685$ & 162.60 \\
\hline 8 & 6 & 100 & $\mathbf{8 , 0 3 0 , 9 8 1}$ & 154.75 & $8,104,990$ & 129.86 \\
\hline 9 & 6 & 100 & $9,213,729$ & 50.34 & $8,104,990$ & 122.13 \\
\hline 10 & 6 & 100 & $8,181,479$ & 77.75 & $\mathbf{8 , 0 3 0 , 9 8 1}$ & 136.61 \\
\hline \multicolumn{2}{|c|}{ Average } & $8,503,426$ & 94.04 & $8,261,793$ & 140.07 \\
\hline
\end{tabular}

Table 6: Results for test network 2 when nodes are randomly ordered 
exploration strategy. Using this strategy, the mean deviations from the optimal for the delta-change and Tabu Search are respectively of $5.9 \%$ and $2.9 \%$.

The following conclusions can be done from the two small networks:

- We notice that on both tests, the delta change allows to significantly reduce the total costs of investment of the network (approximately $7 \%$ for test 1 and $18 \%$ for test 2 with the determinist exploration strategy) which justifies the use of this tool to design the network.

- Both algorithms have obtained the optimal solution when the nodes were randomly explored and for both small networks. And both algorithms failed to identify the optimal solution when the nodes were explored using the deterministic strategy and that for both small networks.

- When nodes are randomly browsed, Tabu search algorithm shows on average better performances compared to the delta-change algorithm. However, the costs differences are very small.

- The deviation percentages of the near-optimal solutions with regards to the optimal solutions are bounded within the $5 \%$ range for both heuristics.

- The computational times savings achieved using heuristics methods to identify the optimal solution is significant (between 64s to 182s using heuristics instead of roughly 50,000s using enumerative methods).

Furthermore, contrary to the intuition collectively accepted, the decrease in cost is made in two cases while the total length of the proposed network increases of $5 \mathrm{~km}$ on test 1 and more than $30 \mathrm{~km}$ on test 2 . Hence, the decrease of the costs results only from the decline of the proposed diameters. Moreover, the topology of the optimal networks is not known in advance. Indeed, the topology obtained with the delta change by starting from the same minimal spanning tree is strongly different in case 1 and case 2 (trees after delta change shown on Figure 2.b and Figure 3.b have only 3 shared arcs out of 6 arcs).

\subsection{Realistic urban network}

We present here the results obtained on a realistic network of existing refueling stations in a French city accommodating not only regular gasoline but also hydrogen delivery. It contains 81 nodes including a unique source. The optimal solution is not reachable within reasonable computation times and it is not feasible to use an exact method since there are $\left(81^{79}\right)$ trees to be explored according to Cayley's formula. Therefore, we used the heuristic methods to overcome this hurdle.

The initialization of the sizing algorithm is made in the following manner. First, we determine a minimal spanning tree. Then, using the sizing module, we obtain diameters included between 20 and $292 \mathrm{~mm}$ by respecting the constraints of minimal and maximal imposed pressures, in the range of 36 and 71 bar (identical to those of Yang et al [37]). The cost associated to this initial network is $25,337,207 €$. 
Table 7 presents the results for the real network when nodes are ordered from the closest to the furthest.

\begin{tabular}{|c|c|c|c|c|c|}
\hline Visited & \% of invest. & \multicolumn{2}{|c|}{ DC } & \multicolumn{2}{c|}{ TS } \\
\cline { 3 - 6 } nodes $n_{i, j}$ & nodes $n_{i}$ & Cost $(€)$ & CPU (s) & Cost $(€)$ & CPU (s) \\
\hline 2 & 5 & $25,337,207$ & 106.75 & $25,337,207$ & 159.29 \\
\hline 3 & 5 & $25,337,207$ & 149 & $25,337,207$ & 167.38 \\
\hline 4 & 5 & $24,785,647$ & 225.46 & $24,785,647$ & 348.27 \\
\hline 5 & 5 & $24,225,351$ & 238.15 & $24,225,351$ & 414.64 \\
\hline 6 & 5 & $24,225,351$ & 266.79 & $24,225,351$ & 403.99 \\
\hline \multicolumn{2}{|c|}{ Average } & $24,782,153$ & 197.23 & $24,782,153$ & 298.71 \\
\hline 2 & 10 & $24,216,507$ & 200.10 & $24,214,899$ & 288.02 \\
\hline 3 & 10 & $24,216,507$ & 274.86 & $25,327,013$ & 429.95 \\
\hline 4 & 10 & $24,448,559$ & 438.63 & $24,785,647$ & 438.16 \\
\hline 5 & 10 & $24,194,827$ & 498.59 & $24,225,351$ & 414.48 \\
\hline 6 & 10 & $23,983,781$ & 645.4 & $24,225,351$ & 416.45 \\
\hline \multicolumn{2}{|c|}{ Average } & $24,212,036$ & 411.52 & $24,555,652$ & 397.41 \\
\hline 2 & 50 & $24,092,891$ & 1054.43 & $\mathbf{2 2 , 7 4 9 , 9 9 2}$ & 492.67 \\
\hline 3 & 50 & $23,583,770$ & 1085.30 & $25,327,013$ & 426.874 \\
\hline 4 & 50 & $23,673,283$ & 1643.80 & $24,785,647$ & 435 \\
\hline 5 & 50 & $23,337,936$ & 2042.88 & $24,225,351$ & 417 \\
\hline 6 & 50 & $22,899,145$ & 2892.68 & $24,225,351$ & 406 \\
\hline \multicolumn{2}{|c|}{ Average } & $23,517,405$ & 1743.80 & $24,262,671$ & 435.51 \\
\hline 2 & 100 & $23,937,386$ & 1758.15 & $22,749,992$ & 488.95 \\
\hline 3 & 100 & $\mathbf{2 1 , 4 8 8 , 4 5 3}$ & 2874.08 & $25,327,013$ & 429.98 \\
\hline 4 & 100 & $23,087,430$ & 4341.03 & $24,785,647$ & 438.77 \\
\hline 5 & 100 & $22,640,800$ & 5128.43 & $24,225,351$ & 418.79 \\
\hline 6 & 100 & $22,899,145$ & 6023.41 & $24,225,351$ & 401.25 \\
\hline \multicolumn{2}{|c|}{ Average } & $22,810,643$ & 4025.02 & $24,262,671$ & 435.55 \\
\hline
\end{tabular}

Table 7: Results for realistic urban network when nodes are ordered from the closest to the furthest

Using the determinist exploration strategy, the best solution identified by deltachange has a cost of $21,488,453 €$ while the best one identified by Tabu search has a cost of $22,749,992 €$ (about $5.8 \%$ more expensive). Performances of both algorithms are the same for a very low $(5 \%)$ percentage of explored nodes. For all other rates of exploration, average values indicate that the delta-change algorithm is better than Tabu search. Average computational times indicate that the Tabu search was faster than delta-change except for the smallest exploration rate (5\%).

Table 8 summarizes the results for the real network when nodes are randomly ordered. Using this strategy, each simulation was repeated 5 times. Statistical indicators were computed from all repetitions and only the mean value was given in table $8^{1}$. The best solution identified by delta-change has a cost of $21,912,261 €(2$ neighbors and $100 \%$ of the investigated nodes $)$ while the best one identified by Tabu search has a cost of $23,324,526 €(6$ neighbors and $10 \%$ of

\footnotetext{
${ }^{1}$ The other data are not shown but could be supplied on simple request
} 
the investigated nodes). The Tabu search best solution was about $6.4 \%$ more expensive than the delta-change one.

\begin{tabular}{|c|c|c|c|c|c|}
\hline Visited & \% of invest. & \multicolumn{2}{|c|}{ DC } & \multicolumn{2}{c|}{ TS } \\
\cline { 3 - 6 } nodes $n_{i, j}$ & nodes $n_{i}$ & Cost $(€)$ & CPU (s) & Cost $(€)$ & CPU (s) \\
\hline 2 & 5 & $25,137,500$ & 73.57 & $24,899,476$ & 192.89 \\
\hline 3 & 5 & $25,202,798$ & 85.52 & $24,923,763$ & 280.17 \\
\hline 4 & 5 & $25,131,708$ & 145.65 & $24,366,149$ & 373.58 \\
\hline 5 & 5 & $24,863,301$ & 179.55 & $25,038,583$ & 494.93 \\
\hline 6 & 5 & $25,023,396$ & 222.42 & $24,963,025$ & 520.50 \\
\hline \multicolumn{2}{|c|}{ Average } & $25,071,740$ & 141.34 & $\mathbf{2 4 , 8 3 8 , 1 9 9}$ & 372.41 \\
\hline 2 & 10 & $25,116,878$ & 144.63 & $24,858,101$ & 327.83 \\
\hline 3 & 10 & $25,220,287$ & 234.28 & $24,568,152$ & 413.1 \\
\hline 4 & 10 & $24,924,057$ & 235.32 & $24,865,503$ & 422.75 \\
\hline 5 & 10 & $24,632,543$ & 279.44 & $24,955,175$ & 463.60 \\
\hline 6 & 10 & $24,664,889$ & 345.22 & $24,387,538$ & 570.79 \\
\hline \multicolumn{2}{|c|}{ Average } & $24,911,731$ & 247.78 & $\mathbf{2 4 , 7 2 6 , 8 9 4}$ & 439.61 \\
\hline 2 & 50 & $23,913,050$ & 745.40 & $24,611,745$ & 493 \\
\hline 3 & 50 & $23,843,802$ & 1053.28 & $24,658,094$ & 478.29 \\
\hline 4 & 50 & $23,437,594$ & 1542.12 & $24,506,438$ & 490.46 \\
\hline 5 & 50 & $23,273,523$ & 1820.38 & $24,554,546$ & 497.96 \\
\hline 6 & 50 & $23,437,558$ & 2142.02 & $24,607,734$ & 494.51 \\
\hline \multicolumn{2}{|c|}{ Average } & $\mathbf{2 3 , 5 8 1 , 1 0 5}$ & 1460.64 & $24,587,711$ & 490.85 \\
\hline 2 & 100 & $23,012,366$ & 1689.80 & $24,210,668$ & 469.46 \\
\hline 3 & 100 & $23,337,715$ & 2528.42 & $24,628,402$ & 482.99 \\
\hline 4 & 100 & $23,030,474$ & 3085.76 & $24,623,527$ & 476.06 \\
\hline 5 & 100 & $23,199,455$ & 4215.36 & $24,440,358$ & 506.40 \\
\hline 6 & 100 & $22,579,375$ & 5046.70 & $24,929,939$ & 513.60 \\
\hline \multicolumn{2}{|c|}{ Average } & $\mathbf{2 3 , 0 3 1 , 8 7 7}$ & 3313.21 & $24,566,579$ & 489.70 \\
\hline
\end{tabular}

Table 8: Results (mean values) for the realistic urban network when nodes are randomly ordered

The following conclusions can be done from the real network example:

- Substantial gains are obtained with regard to the cost of the initial minimal spanning tree (up to $16 \%$ saved using the delta-change with determinist exploration).

- The network cost significantly decreases with the number of tested nodes $n_{i}$, and slowly decreases with the number of not directly connected nodes $n_{i, j}$ visited in the neighbors of $n_{i}$.

- The delta-change computation time linearly increases with the number of tested nodes $n_{i}$ and with the number nodes $n_{i, j}$ visited in the neighbors of this node. The Tabu search computational time is less sensitive to these two parameters.

- Based on the average values' comparison, the performances of the two used algorithms are roughly the same. However, considering only the best solutions identified by the delta-change and Tabu search algorithms shows that 
there is a relative superiority of the delta-change.

- Even if the delta-change doesn't outperform the Tabu search in all cases, we noticed that using the random exploration strategy improves its performances compared to those of Tabu search.

- It seems that the random choice of the node can produce variable costs. On the other hand, the ordered choice of nodes $n_{i}$ implies costs strictly decreasing with the number of node $n_{i}$ and with the number of node $n_{i, j}$ explored. This phenomenon can be explained by the fact that savings are obtained on nodes closest to the source and that it is not worth continuing more downstream. The random choice of node $n_{i}$ gives slightly better solution when considering $100 \%$ of the nodes $n_{i}$ explored.

From the results presented in Tables 7 and 8 and based on our experiences, we recommend to use the delta-change to randomly and extensively explore the network $\left(100 \%\right.$ of the node $n_{i}$ ) with a combination of only 2 to 3 neighbors $\left(n_{i, j}\right)$. Hopefully, this recommendations could allow us to obtain solutions which are better than those identified using the determinist strategy.

\subsection{Application to a new national network in France}

\subsubsection{Delta Change versus Minimal length spanning tree}

We tested the method presented in the previous sections on the France test case by selecting the current urban areas with more than 100,000 inhabitants (78 as of the year 2000). The demand for hydrogen will be estimated for each city in France based on:

- A full conversion of the car engines from gasoline to hydrogen i.e. a market share of $100 \%$ for hydrogen as a fuel (for an horizon beyond 2050).

- An average consumption of hydrogen per habitant based on the current consumption of regular and diesel gasolines.

- The current population of each city as of 2007 (no increase of population has been taken into account).

As a first step, we consider one central production plant for the whole of France located near Paris with an inlet pressure of 100 bars and a minimum required pressure of 35 bars without any intermediate compression stations. Then, we apply and compare the 2 approaches:

- Minimal length spanning tree followed by the optimization of the diameters (Figure 4).

- The algorithm of delta change combining the test of the optimal diameter at each new topology (Figure 5).

The following conclusions can be exhibited:

- The national network is defined to link main French cities with the network layout follows some natural corridors: Rhone's valley, Paris-Bordeaux, 


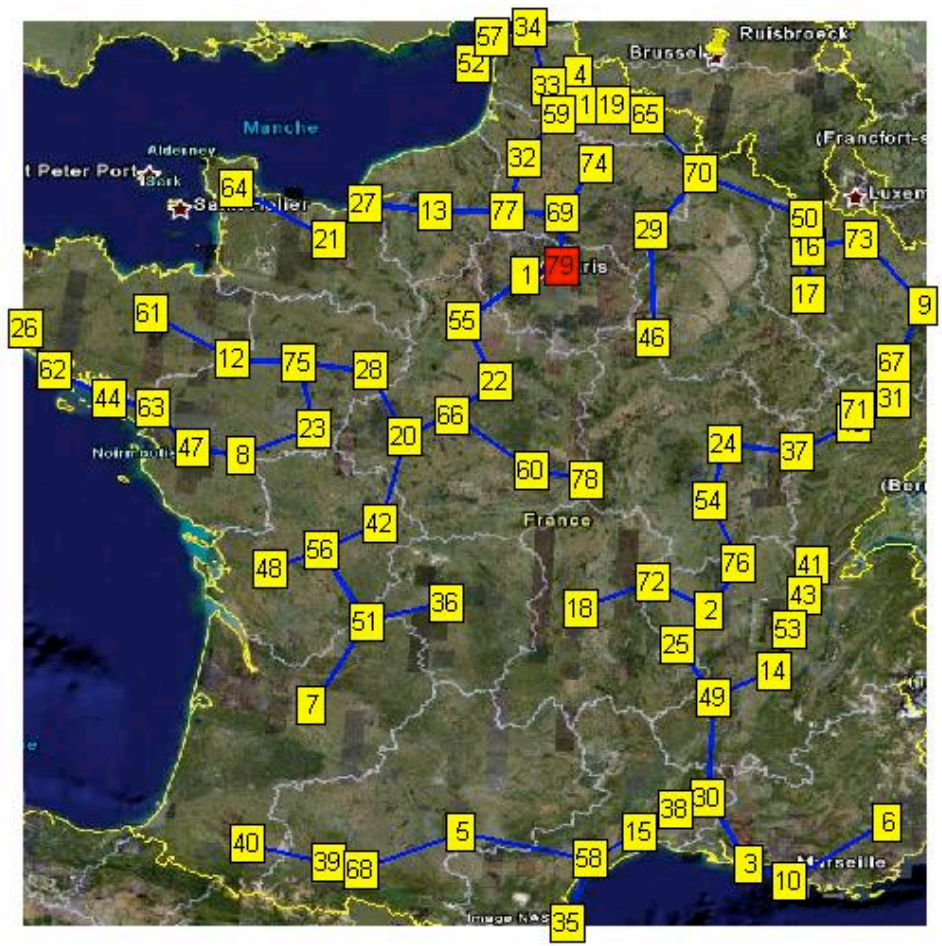

Figure 4: French network with minimal length spanning tree 


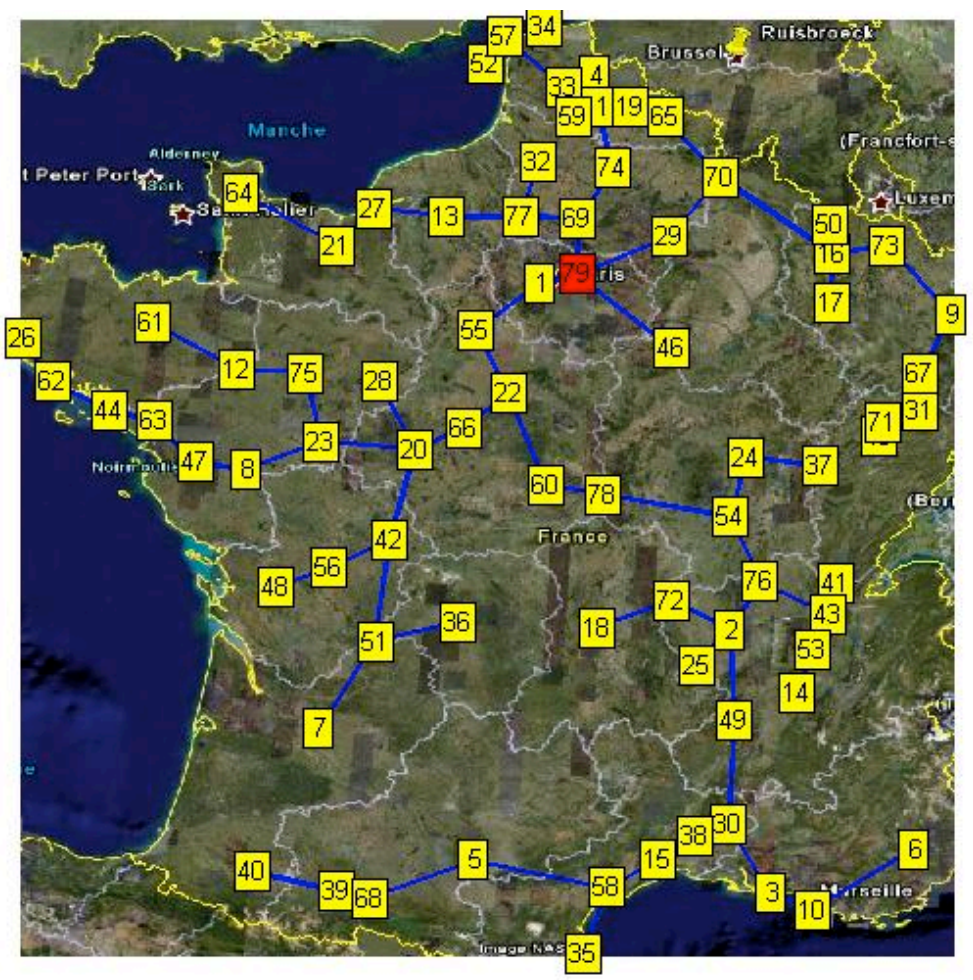

Figure 5: Proposed French national hydrogen network with delta change 
French riviera, etc. Overall, the network looks like the national natural gas network.

- The minimal length spanning tree is $5,035 \mathrm{~km}$ long while the length of the delta change network is longer with $5,274 \mathrm{~km}(+4.7 \%)$.

- The Delta Change significantly modified the branches of the initial minimal spanning tree network. Let us note in particular the connection between Paris and the South-East part of France is done through the center of France with the delta change instead of the East with the minimal length spanning tree. The significant difference between the Delta change solution and the minimal length spanning tree is the link between node 78 and node 54 .

- Even if the diameters of both networks are in the same range [70-1000] mm, the average value of diameters dropped from $440 \mathrm{~mm}$ to $300 \mathrm{~mm}$.

- The total investment cost dramatically decreased by $18 \%$ to 2.34 7 $€$ billion from the Delta change solution compared to $2.868 €$ billion initially with the minimal length spanning tree.

\subsubsection{Impact of the location of the hydrogen central plant}

In this case, we analyze the impact of the change of location for the central plant from Paris (which can be considered as a rough approximation of the weighted center of the consumption nodes) to a node far from the consumption areas.

Let us first notice that:

- The overall structure of the network has not changed with the same main streamlines.

- The total investment costs dramatically increased from 2.347 b $€$ to 3.194 $b €(+36 \%)$.

We can then conclude that the plant location has a tremendous impact on the investment costs since large quantities of hydrogen have to be carried over longer distances. Therefore, it makes sense to locate the plants nearby the main consumption urban areas.

\subsubsection{Impact of national versus regional hydrogen production plants}

The network designed with one sourcing node implies large quantities transported over long distances. However, unlike natural gas that is imported from sources outside of France, the hydrogen may be locally produced at different locations on the mainland. This part is dedicated to assess the impact of such breakdown on the investment costs for the pipeline network compared to the single source pipeline network.

Therefore, we propose to split the national production into 4 regional production centers with 4 attached pipeline networks: the North, South, West and East. The selection of the cities belonging to each of these zones has been made based on considerations of balancing quantities to be delivered (except for Paris that 


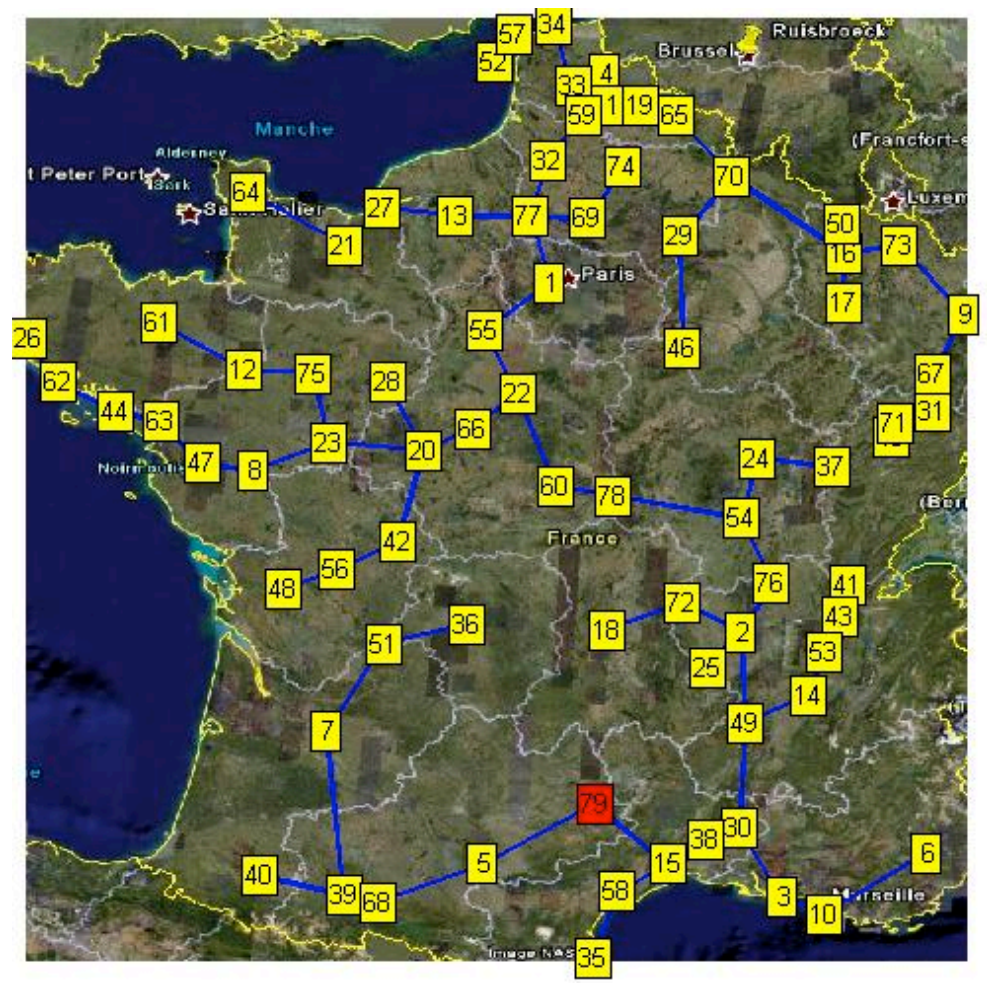

Figure 6: Network with a central plant located far from the consumption nodes 
is over-represented) but has not been part of the optimization (pre-process). To be able to have the same comparison basis as in the single plant case, we kept the injection pressure at 100 bar and the delivery points higher than 35 bar. The Delta change method is applied to each of this test case.

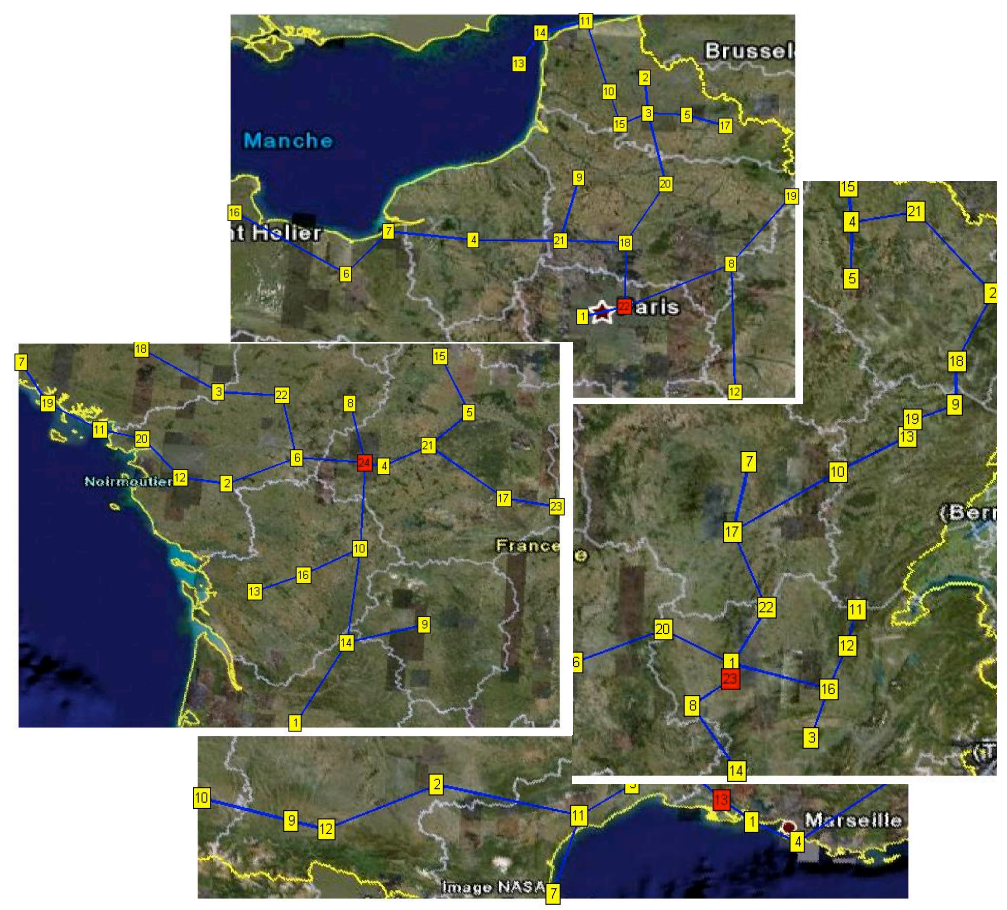

Figure 7: Proposed four Regional network topologies

\begin{tabular}{|l|c|c|c|c|}
\hline Network & $\begin{array}{c}\text { Transported } \\
\text { quantities } \\
(\mathrm{kg} / \text { year })\end{array}$ & $\begin{array}{c}\text { Investment } \\
\text { costs }(€)\end{array}$ & $\begin{array}{c}\text { Length } \\
(\mathrm{km})\end{array}$ & $\begin{array}{c}\text { Av. diameter } \\
(\mathrm{mm})\end{array}$ \\
\hline North & $1,615,572,826$ & $395,790,406$ & 1,202 & 210 \\
West & $579,969,519$ & $503,614,131$ & 1,588 & 186 \\
East & $661,510,358$ & $440,690,984$ & 1,208 & 255 \\
South & $565,190,102$ & $311,303,953$ & 890 & 240 \\
TOTAL & $3,422,242,805$ & $1,651,399,474$ & 4,889 & $\mathrm{~N} / \mathrm{A}$ \\
National & $3,422,242,805$ & $2,342,722,894$ & 5,274 & 307 \\
\hline
\end{tabular}

Table 9: Comparison of features of each of the regional network versus the national network

We can see that the option to have several regional networks drives the investment costs down compared to the national network (-30\%) due to the following reasons:

- The total cumulative length of all the regional networks is shorter than the national network $(4,889$ instead of $5,274 \mathrm{~km})$ and this factor accounts for $20 \%$ of the cost reduction.

- The regional networks have lower pipe diameters than the diameters on the 
national network and this effect accounts for $80 \%$ of the cost reduction.

\section{Discussions on network deployment}

Beyond the network design and sizing, we plan as well to investigate the time phasing of the deployment of these pipeline networks. This time phasing is usually critical to spread over time the investment but in this case, the benefits of time phasing can be assessed with regards to alternate transportation modes. As a matter of fact, even if the choice of the pipeline may make sense over the longterm with enough high throughput in the pipes and for enough long distances, the choice of the pipeline can be challenged at lower levels of demand. At early stages of development of the hydrogen supply chain, the delivery by trucks brings the combined advantages of low initial investment costs and the flexibility to deliver small and infrequent amounts of hydrogen. However, the supply chains by trucks have to rely on significant investments on liquefiers (for the cryogenic option) or compressor stations (to fill the trailers) that might be significant with long term payback periods. Then, based on market share evolutions, the pipeline CAPEX and OPEX costs will be compared at each decision time steps to the corresponding costs on the truck side to determine the time to switch from the trucks to the pipeline option. In this competition between transportation modes, the tradeoff will have to be found as soon as the economy of scale provided by the pipeline with enough high rates compensates for the flexibility given by the truck deliveries.

Although a part of the investments related to the tractors and the drivers won't be impacted by the hydrogen's state, a significant part of these investments (trailers and the upstream and downstream equipments) will depend on the phase of hydrogen: gaseous or liquid. Let us remind that in all cases the production will come from a central plant.

- In the gaseous case, unlike for the pipeline option which requires low compression power at the central plant (injection around 60 bars) but high booster compression rates at the refueling stations (injection between 450 and 700 bars in the car's tank), the gas trailer will require significant amount of compression at the central plant to feed the trailer (at up to 400 bars) but limited compression on the refueling site will be necessary (only for tanks higher than 400 bars).

- In the liquid case, the main additional investment consists of the liquefaction unit located right after the central plant. Besides, the liquid hydrogen will need dedicated cryogenic tanks both at the production site and at the delivery station as well as on the truck with cryogenic trailers. As we can consider the liquid tanks inside the cars as too costly, delivery to cars will be under gaseous form. Hence, vaporization will occur and booster compression will be necessary to reach the desired pressure level in the gaseous cars tanks.

This deployment problem belongs to the large class of multistage network design problem (Triadou [34], Kubat [22], Yi [38], Olorunniwo and Jensen [26]). The goal of all these approaches is to delay at the latest stage the required investment 
to match the progressive levels of demand (the preference for paying later is represented with the use of a discount factor on future costs). The specificity of the hydrogen supply chain lies in the fact that the pipelines compete with other hydrogen carriers: the compressed gas trucks and the liquid cryogenic trucks.

The economic decision criterion will be based on the computation of the average yearly cost of the equipment from the net discounted cost based on initial capital expenditure (CAPEX) discounted over the lifetime of the assets and an operational expenditure (OPEX). This yearly average cost will be computed for each section of the different considered supply chain. Then each cost will be added to be able to compare the 3 supply chains between them.

Therefore, based on assumptions made for the truck deliveries (position of the trucks depot close or not to the plant, roundtrip loops, multiple deliveries per loop, assignments of trucks to customers...), the optimal choice of delivery mode will be a function of distance and flow rate. The delivery costs by trucks or pipelines will increase with the distance from the central plant but not at the same rate. The pipeline delivery costs will be higher than the delivery by truck for the nearest points to the source and lower than the delivery costs by truck for the furthest points to the source. Besides, the flow rate factor will need to be considered in the choices. Over short distances gaseous truck delivery will be optimal especially at low flow rates but pipelines may be optimal at higher flow rates. Economies of scale will be visible over long distance pipeline connecting several customers with cheaper marginal costs to reach a new customer from the last served customers by an existing pipeline system.

\section{Conclusions and perspectives}

In the context of the scarcity of petroleum products as fuels, hydrogen appears as a promising solution that respects the environment for which it is already necessary to define the relevant transportation system. In this paper, we presented a new methodology for the simultaneous determination of the topology and of the diameters of hydrogen transport networks. These two problems were generally solved separately. For example, the determination of the optimal topology is generally based on some basic criteria such as the minimum length of the network. Our solution method was tested on a network of refueling gas stations in a middle size French city as well as at the regional and national levels. This study shows that the 2 stage-approach generally used (first looking for a network topology of minimal length and then optimizing the diameters for this fixed topology) is not sufficient. On the contrary, our case studies showed that increasing the total length of the network can help to decrease the network cost by using smaller diameters for some pipes. The software developed in this work allows many other functionalities as added value compared to other available tools. For sake of clarity, many of these functionalities were not presented in this paper. We plan to consider in a near future the network temporal deployment more deeply. The optimal facility location/allocation problem for multi-source (i.e many hydrogen production plants) network will also be studied. Finally, in order to evaluate the genericity of the software, we shall study other geographies (towns, countries, ...). 


\section{Acknowledgments}

This research was done with the financial support of the National Research Agency ANR (Agence Nationale de la Recherche). The projet research, named ECOTRANSHY for Economy of Transport for Hydrogen, is a projet of the research program PAN-H for Programme d'Action Nationale pour l'Hydrogène. We would like to thank participants at various seminars and conferences for insightful comments as well as Gino Fortuni.

\section{References}

[1] Abd-El-Barr, M. (2009). Topological network design: A survey. Journal of Network and Computer Applications, 32(3), 501-509.

[2] André J., Bonnans F., Cornibert L., Optimization of capacity expansion planning for gas transportation networks, European Journal of Operational Research, Volume 197, Issue 3, Pages 1019-1027, September 2009.

[3] Babonneau F., Nesterov Y., Vial J.Ph., Design and operations of gas transmission networks, CORE DP 2009/48, Louvain-La-Neuve, Belgium to appear in Operations Research, 2009.

[4] Ball M., Wietschel M., Rentz O. Integration of a hydrogen economy into the German energy system: an optimizing modelling approach, International Journal of Hydrogen Energy, Volume 32, Issues 10-11, July-August 2007, Pages 1355-1368.

[5] Bang Ye Wu, Kun-Mao Chao, Spanning Trees and Optimization Problems, Chapman \& Hall/CRC Press, USA, 2004.

[6] D.P. Bertsekas. Dynamic programming and Optimal Control, Second Edition, Vol. 1. Massachussetts Institute of Technology. Athena Scientific, 2000.

[7] Bhaskaran S., Salzborn F., 1979. Optimal design of gas pipeline networks. Journal of the operations research society, 30, pp.1047-1060.

[8] Brimberg J., P. Hansen, K.-W. Lin, N. Mladenovic, and M. Breton, An Oil Pipeline Design Problem Operations Research 51, March/April 2003, pp 228239.

[9] Castello P., Tzimas E., Moretto P. and Paeteves S.D., 2005. Techno-economic assessment of hydrogen transmission and distribution systems in Europe in the medium and long term, French Commission, Joint Research Center.

[10] de la Cruz, J. M., de Andres-Toro, B., Herran, A., Porta, E. B., and Blanco, P. F. (2003, 16-19 Sept. 2003). Multiobjective optimization of the transport in oil pipelines networks. Paper presented at the Emerging Technologies and Factory Automation, 2003. Proceedings of ETFA '03. IEEE Conference.

[11] De Wolf D, Smeers Y. Optimal dimensioning of Pipe Networks with application to Gas Transmission Network. Operations Research, 44(4):596-608, 1996. 
[12] Didi Biha M., Kerivin H., Mahjoub A.R., Steiner Trees and polyhedra, Discrete Applied Mathematics 112 (2001) 101-120.

[13] Dolan, A., Aldous J., Networks and Algorithms, an Introductory Approach, Ed. John Wiley \& Sons, 1995.

[14] E.K. Donkoh, S.K. Amponsah and K.F. Darkwah(2011). Optimal Pipeline Connection for the West African Gas Pipeline Project. Research Journal of Applied Sciences, Engineering and Technology 3(2): 67-73, 2011.

[15] Gill P., Murray W., Saunders M.A., 2005, SNOPT:An SQP Algorithm for Large-Scale Constrained Optimization, SIAM Review, Vol. 47, No.1, pp. 99131.

[16] Gouveia, L., Simonetti, L., Uchoa, E. (2011). Modeling hop-constrained and diameter-constrained minimum spanning tree problems as Steiner tree problems over layered graphs. Mathematical Programming, 128(1), 123-148.

[17] Hafner M., 1994. Gaz naturel et production d'électricité : analyse technologique et économique de la génération d'électricité et du transport de gaz pour les pays du bassin méditerranéen. Thèse de doctorat, Ed. Technip.

[18] HyFrance 2 project, Final Report: Evaluation technico-économique du développement dune filière hydrogêne en France et de ses impacts sur le système énergétique, Economie et Environnement, September 2007, http://www.ecolo.org/documents/documents in french/H2-rapportHyFrance 07.pdf

[19] Johnson N. and J. Ogden, A spatially-explicit optimization model for longterm hydrogen pipeline planning, international journal of hydrogen energy 37 (2012) pp 5421- 5433.

[20] Joulié R., 1998. Mécanique des fluides appliquée. Editions Ellipses.

[21] Kerivin H. and Mahjoub A. R. (2005). Design of Survivable Networks: A survey. Netw., 46(1), 1-21.

[22] P. Kubat, J. MaxGregor Smith. A multi-period network design problem for cellular telecommunication systems. European Journal of Operational Research 134, 439-456, 2001.

[23] Lin, Fan, Ogden, Sperling, The Hydrogen Infrastructure Transition Model (HIT) \& Its Application in Optimizing a 50-year Hydrogen Infrastructure for Urban Beijing, UCD-ITS-RR-06-05, 2005.

[24] Kapoor, S., Ramesh, H. 1991. Algorithms for generating all spanning trees of undirected, directed and weighted graphs. Algorithms and Data Structures. In F. Dehne, J.-R. Sack, N. Santoro (Eds.), Vol. 519, pp. 461-472. Springer Berlin / Heidelberg.

[25] R.S. Middleton and J.M. Bielicki, A scalable infrastructure model for carbon capture and storage: SimCCS. Energy Policy, 37 (2009), pp. 1052-1060.

[26] F. O. Olorunniwo, P.A. Jensen. Optimal Capacity Expansion policy for natural Gas transmission networks-A decomposition approach. Engineering Optimization, Vol. 6, pp. 13-30, 1982 
[27] Minty, G. 1965. A Simple Algorithm for Listing All the Trees of a Graph. Circuit Theory, IEEE Transactions on, 12(1) , 120-120.

[28] Osiadacz AJ, Gorecki M. Optimization of Pipe Sizes for Distribution Gas Network Design. PSIG Conference, 27th Annual Meeting, October 1995.

[29] Pandit A. and Crittenden J.C. Index of Network Resilience (INR) for Urban Water Distribution Systems, Critical Infrastructure Symposium, April 23-24 2012, Arlington, VA, USA.

[30] Parker N., 2005. Using Natural gas Transmission Pipeline Costs to Estimate Hydrogen Pipeline Costs, Technical report No. UCD-ITS-RR-04-3, Institute of Transportation Studies, University of California, Davis.

[31] Patay E., Ghaffari A., Minoux M., Perrin J., Surrogate approximation for the cost evaluation of infrastructure development for hydrogen as an automotive fuel, Proceedings 19th Mini EURO Conference on the Energy Sector, ORMMES 06, 6-9 September 2006.

[32] Rothfarb B., Frank H., Rosenbaum D.M., Steiglitz K and Kleitman D.J., 1970. Optimal design of offshore natural-gas pipeline systems. Operations research,18(6), p. 992-1020.

[33] Smit R., Weeda M., De Groot A. Hydrogen infrastructure development in The Netherlands, International Journal of Hydrogen Energy 32 (2007) 13871395 .

[34] C. Triadou. Dimensionnement de réseau multipériode et relaxation Lagrangienne. Actes du Congrés ROADEF, 2003.

[35] Nie Ting Ze, Optimal Lay-Out of Natural Gas Pipeline Network, 23rd World Gas Conference, Amsterdam, 2006.

[36] Walters G.A., 1987. Dynamic programming approach to the optimal design of tree-like networks. Proceedings of the international conference on Optimization Techniques and Applications, Singapour, Singapore, p. 487-496.

[37] Yang C. \& Ogden J., 2007. Determining the lowest-cost hydrogen delivery mode. International Journal of Hydrogen Energy, 32(2), p. 268-286.

[38] F. Yi. Capacity planning for a natural gas pipeline in Sweden. Energy Economics, p. 211-215, July 1990.

[39] Zhang J, Zhu D. A Bilevel Programming Method for Pipe Network Optimization. SIAM Journal on Optimization, 6(3):838-857, 1996. 


\section{Appendix}

\subsection{Characteristics of the optimal topology}

We demonstrate here that with the choice made within the framework of the ECOTRANSHY project for the investment objective function and for the head losses equation, the optimal networks are trees by using the following result of Bhaskaran and al. [7]:

Lemma. Considering the following head losses equation

$$
D_{i j}=N Q_{i j}^{\beta_{1}}\left(\frac{L_{i j}}{\pi_{i}-\pi_{j}}\right)^{\beta_{3}}
$$

and the following investment objective function:

$$
\min C O S T=\sum_{(i, j) \in A} L_{i j} C\left(D_{i j}\right) .
$$

If the following condition is satisfied

$$
D_{i j} \frac{C^{\prime \prime}\left(D_{i j}\right)}{C^{\prime}\left(D_{i j}\right)}<\frac{1-\beta_{1}}{\beta_{1}}
$$

then the optimal network is a tree.

Proof: See Bhaskaran et Salzborn [7].

The objective function for investment chosen in the ECOTRANSHY project is the following:

$$
C\left(D_{i j}\right)=a_{0}+a_{1} D_{i j}+a_{2} D_{i j}^{2}
$$

The head losses equation used in the ECOTRANSHY is the following:

$$
\pi_{i}-\pi_{j}=k^{\prime} \frac{L_{i j} Q_{i j}^{2}}{D_{i j}^{5}} .
$$

Solving this equation for $D_{i j}$, we obtain:

$$
D_{i j}=k^{\prime \prime} Q_{i j}^{\frac{2}{5}}\left(\frac{L_{i j}}{\pi_{i}-\pi_{j}}\right)^{\frac{1}{5}}
$$

By comparison with (6), we can conclude that: $\beta_{1}=\frac{2}{5}$. Compute now the first and second derivatives of our investment objective function:

$$
\begin{aligned}
C^{\prime}\left(D_{i j}\right) & =2 a_{2} D_{i j}+a_{1} \\
C^{\prime \prime}\left(D_{i j}\right) & =2 a_{2}
\end{aligned}
$$

Compute now:

$$
D_{i j} \frac{C^{\prime \prime}\left(D_{i j}\right)}{C^{\prime}\left(D_{i j}\right)}=D_{i j} \frac{2 a_{2}}{2 a_{2} D_{i j}+a_{1}}=\frac{2 a_{2} D_{i j}}{2 a_{2} D_{i j}+a_{1}}
$$

Let us consider now the two following cases: 
Case 1. $a_{1}=\mathbf{0}$. In this case, condition (8) becomes:

$$
D_{i j} \frac{C^{\prime \prime}\left(D_{i j}\right)}{C^{\prime}\left(D_{i j}\right)}=\frac{2 a_{2} D_{i j}}{2 a_{2} D_{i j}}=1<\frac{1-\beta_{1}}{\beta_{1}}
$$

What is equivalent to say that:

$$
\beta_{1}<1-\beta_{1} \Leftrightarrow \beta_{1}<\frac{1}{2}
$$

In our case, we have seen here above that $\beta_{1}=\frac{2}{5}$. The condition is thus satisfied.

Case 2. $a_{1}>0$. In this case, the condition (8) becomes :

$$
D_{i j} \frac{C^{\prime \prime}\left(D_{i j}\right)}{C^{\prime}\left(D_{i j}\right)}=\frac{2 a_{2} D_{i j}}{2 a_{2} D_{i j}+a_{1}}=\epsilon<\frac{1-\beta_{1}}{\beta_{1}}
$$

with $\epsilon \in] 0,1[$. What is equivalent to:

$$
\epsilon \beta_{1}<1-\beta_{1}
$$

or:

$$
\beta_{1}(\epsilon+1)<1 \Leftrightarrow \beta_{1}<\frac{1}{\epsilon+1}
$$

Examine the two limit cases:

$$
\begin{array}{lll}
\epsilon=0 & \text { the condition becomes } & \beta_{1}<1 \\
\epsilon=1 & \text { the condition became } & \beta_{1}<\frac{1}{2}
\end{array}
$$

Thus, for all $\epsilon \in] 0,1\left[\right.$, if $\beta_{1}<\frac{1}{2}$, the condition is satisfied. In our case, $\beta_{1}=\frac{2}{5}$. The condition is thus satisfied.

This completes the proof. 